\title{
Genomes of Wolbachia endosymbionts from the human filarial parasites Mansonella perstans and Mansonella ozzardi
}

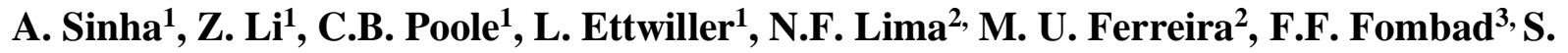 \\ Wanji $^{3}$ and Clotilde K. S. Carlow ${ }^{*}$
${ }^{1}$ New England Biolabs, Ipswich, Massachusetts, 01938, USA; ${ }^{2}$ Department of Parasitology, Institute of Biomedical Sciences; University of São Paulo, São Paulo, Brazil; ${ }^{3}$ Department of Microbiology and Parasitology, University of Buea, Buea, Cameroon

Running Title: Genomes of Wolbachia from Mansonella spp.

*Author for correspondence: Clotilde Carlow, Division of Genome Biology, New England Biolabs, Ipswich, MA 01938. Tel: 978380 7263, Fax: 978921 1350, e-mail: carlow@neb.com.

Data deposition: Raw Illumina reads for the $M$. perstans isolate Mpe1 and the $M$. ozzardi isolate Moz2 have been deposited in the NCBI SRA database under BioProject accession numbers PRJNA666672 and PRJNA666671 respectively. The assembled genome and PGAP annotations for the Wolbachia $w \mathrm{Mpe}$ and $w \mathrm{Moz}$ are available from the NCBI GenBank database with accession numbers JACZHU000000000 and JADAKK000000000 respectively. 


\section{Abstract}

24 Mansonella ozzardi and Mansonella perstans, filarial parasites infecting millions of people

25 worldwide, harbor their unique obligate endosymbionts, the alpha-proteobacteria Wolbachia

$26 \quad w \mathrm{Moz}$ and $w \mathrm{Mpe}$, respectively. Currently, little is known about these Wolbachia and no genome

27 sequences are available. In the current study, high quality draft genomes of $w \mathrm{Moz}$ and $w \mathrm{Mpe}$

28 were assembled from complex clinical samples using a metagenome assembly and binning

29 approach. These represent the first genomes from supergroup F Wolbachia originating from

30 human parasites and share features characteristic of filarial as well arthropod Wolbachia,

31 consistent with their position in supergroup F. Metagenomic data analysis was also used to

32 estimate Wolbachia titers, which revealed wide variation in levels across different clinical

33 isolates, addressing the contradicting reports on presence or absence of Wolbachia in M.

34 perstans. These findings may have implications for the use antibiotics to treat mansonellosis.

35 The $w \mathrm{Moz}$ and $w \mathrm{Mpe}$ genome sequences provide a valuable resource for further studies on

36 symbiosis, evolution and drug discovery.

37

38

39

40

41 


\section{Introduction}

44 Wolbachia are gram-negative $\alpha$-proteobacteria of the order Rickettsiales, present as intracellular

45

46

47

symbionts in many species of parasitic filarial nematodes and arthropods ${ }^{1}$. While the Wolbachia associations in arthropods range from reproductive parasites to nutritional mutualists ${ }^{1,2}$,

Wolbachia is an obligate mutualist in the filarial nematodes ${ }^{3}$ and is essential for worm

development, fecundity and survival ${ }^{3,4}$. The latest phylogenetic classification, based on Multi-

Locus Sequence Typing (MLST), assigns Wolbachia to various monophyletic supergroups ${ }^{5-7}$.

Supergroups A, B, E, H and S are comprised entirely of arthropod Wolbachia, while supergroups

C, D and J consist of filarial Wolbachia exclusively. Interestingly, the Wolbachia present in the

filarial nematodes of the genus Mansonella are classified under supergroup F, the only

supergroup to have Wolbachia members from nematode as well as arthropod hosts.

Mansonella perstans and Mansonella ozzardi are the two main causes of mansonellosis, and harbor their specific Wolbachia, wMpe and wMoz respectively. Mansonellosis is the most prevalent human filariases, yet the least studied and most neglected of all filarial infections ${ }^{8-13}$.

M. perstans is considered to be the most common filaria in West and Central Africa ${ }^{9,12}$. It is also found in the Amazon rainforest and the Caribbean coast in South America ${ }^{12,14}$. M. ozzardi infections have been reported from many countries in South and Central America, including some Caribbean Islands ${ }^{10,12,15,16}$. Importantly, co-infections of $M$. perstans and $M$. ozzardi have been reported in South America ${ }^{17,18}$, making their treatment challenging as the two species respond differently to the commonly used anti-filarial drugs ${ }^{12}$. Antibiotics targeting Wolbachia have been successful in treating filarial diseases such as onchocerciasis and lymphatic filariasis $^{4,19-24}$. Doxycycline, an effective anti-Wolbachia antibiotic, has been shown to clear $M$. perstans microfilariae ${ }^{12,25-27}$. Although no clinical trials have been reported, it is likley that 
antibiotics will have a similar effect against $M$. ozzardi $^{28}$, enabling the use of a single drug to treat co-infections with $M$. ozzardi and $M . \operatorname{perstan}^{18}$.

There is great interest in understanding the nature of the symbiotic relationship between Mansonella and their Wolbachia endosymbionts. The phylogenetic position of the Mansonella Wolbachia in a unique supergroup that also contains arthropod Wolbachia could also have implications for the nature of interactions with their host $t^{5,6,29,30}$. While genomic information could provide fundamental resource for investigating their biology, the only complete genome from supergroup F Wolbachia is the genome of Wolbachia from the bedbug Cimex lectularius $(w \mathrm{Cle})^{29}$. A draft genome of the supergroup F Wolbachia from Madathamugadia hiepei ( $w$ Mhie), a filarial parasite of geckos, has become available recently ${ }^{6}$. However, since no genomic information is available for the Wolbachia of Mansonella, it is not known whether their biology is more closely aligned to filarial or arthropod Wolbachia ${ }^{12}$.

Here, the first genomes of supergroup F Wolbachia $w \mathrm{Moz}$ and $w \mathrm{Mpe}$ from human filarial parasites are reported. These draft genomes have been assembled from one isolate of $M$. ozzardi microfilariae from Venezuela, and one isolate of $M$. perstans from Cameroon. The genomes are slightly larger than $1 \mathrm{Mb}$ and encode for about 900 genes each. The genome drafts are of high quality based on assessment of completeness using presence of conserved Wolbachia core genes as a metric. Analysis of deep-sequencing data from additional isolates of $M$. ozzardi and $M$. perstans was performed and provided some insights into the longstanding controversy around the status of Wolbachia across different Mansonella perstans isolates ${ }^{25-27,31-33}$. Additionally, comparative analyses between $w \mathrm{Moz}$ and $w \mathrm{Mpe}$, as well as across other filarial and arthropod Wolbachia genomes are also performed and reported. 
The availability of the $w \mathrm{Moz}$ and $w \mathrm{Mpe}$ genome sequences will provide further insight

89 into the evolution and phylogenetic relationships of the Wolbachia endosymbionts of old and

90 new world Mansonella species. They will serve as an important resource for further studies on

91 symbiosis, facilitate the development of improved methods for Wolbachia detection and

92 contribute to the on-going search for new anti-Wolbachia drugs.

\section{Materials and Methods}

\section{Ethics statement}

97 All research involving human subjects was approved by the appropriate committee and performed in accordance with all relevant guidelines and regulations. Informed consent was obtained from all participants or their legal guardians.

For M. perstans, ethical clearance was obtained from the National Institutional Review

101

102

103

104

board, Yaoundé (REF: N²015/09/639/CE/CNERSH/SP) and administrative clearance from the Delegation of Public Health, South-West region of Cameroon (Re: R11/MINSANTE/SWR/ RDPH/PS/259/382). Approval for the study was granted by the "National Ethics Committee of Research for Human Health" in Cameroon. Special consideration was taken to minimize the health risks to which any participant in this study was exposed. The objectives of the study were explained to the consenting donor after which they signed an informed consent form. The participant's documents were given a code to protect the privacy of the study subject. At the end of the study, the donor received a cure of mebendazole (100 mg twice a day for 30 days). 
For M. ozzardi, study protocols were approved by the Institutional Review Board of the

110

111

112

113

Institute of Biomedical Sciences, University of São Paulo, Brazil (1133/CEP, 2013). Written

informed consent was obtained from all patients, or their parents or guardians if participants

were minors aged $<18$ years. Diagnosed infections were treated with a single dose of $0.2 \mathrm{mg} / \mathrm{kg}$

of ivermectin after blood sampling ${ }^{34,35}$.

\section{Parasite materials and DNA extraction}

DNA from M. ozzardi microfilariae from blood samples of individual subjects were collected as part of a previous study in Brazil ${ }^{35}$. One of the high microfilaremia samples was selected for genome sequencing and is denoted as Moz1 in the present study. A Venezuelan isolate of $M$. ozzardi microfilariae was generously donated by Izaskun Petralanda in 1989 and is denoted as Moz2 in this study. Genomic DNA was prepared from Moz2 microfilariae by Proteinase K digestion followed by phenol/chloroform extraction and ethanol precipitation, and drop dialysis

\section{(https://www.neb.com/protocols/2013/09/16/drop-dialysis) then stored at $-20^{\circ} \mathrm{C}$. Two} independent isolates of M. perstans microfilariae, denoted as Mpe1 and Mpe2 in this study, were obtained on nylon filters from blood samples of consenting individuals in Cameroon ${ }^{36}$. Mpe1 DNA was extracted using a Genomic DNA Tissue MicroPrep kit (Zymo Research, USA) as described previously ${ }^{37}$. DNA from Mpe2 was isolated as described above for Moz2. DNA quantity was determined using a Qubit dsDNA HS Assay kit in conjunction with a Qubit 2.0 Fluorometer as directed by the manufacturer (Life Technologies, USA).

\section{Illumina library construction and sequencing}


131 The NEBNext Microbiome DNA enrichment kit (New England Biolabs Inc., USA) was used as

132 directed to enrich for parasite DNA and reduce human DNA contamination prior to construction

133 of the libraries from each of the samples, except Moz2. The preparation of Illumina libraries

134 from Mpe1 and Moz1 samples have been described as part of a previous study ${ }^{37}$. A similar

135 protocol was used for preparing libraries from Mpe2 and Moz2. Briefly, Illumina libraries were

136 constructed using the NEBNext Ultra II FS DNA Library Prep Kit (New England Biolabs Inc.,

137 USA) as described by the manufacturer. Following PCR amplification with different index

138 primers to enable multiplexing, the libraries were size selected using NEBNext Sample

139 Purification Beads (NEB cat. \# E7767) following manufacturer's instructions. The approximate

140 insert size and concentration of each library was determined using a 2100 Bioanalyzer with a

141 high sensitivity DNA chip (Agilent Technologies, USA). Two Mpe2 libraries with insert sizes of

142 approximately 500 and 800 bps and two Moz2 libraries with insert sizes of approximately 500

143 and 950 bps were constructed. Libraries were diluted to $4 \mathrm{nM}$ with $10 \mathrm{mM}$ Tris, $0.1 \mathrm{mM}$ EDTA

$144 \mathrm{pH} 8$ for sequencing. Due to the A:T rich nature of filarial genomes, Phi X DNA was added to

145 balance base pair composition, then sequenced using the Illumina MiSeq and NextSeq500

146 platforms (paired end, $150 \mathrm{bps).}$

148 Metagenomic assembly and binning

149 Raw reads were processed using the BBTools package v38.51 (https://jgi.doe.gov/data-and-

150 tools/bbtools/). Duplicate raw reads and bad tiles were removed using the clumpify.sh and

151 filterbytile.sh utilities. Adapter trimming, removal of phiX reads and reads with 13-nt or longer

152 stretches of the same nucleotide (poly-G, -C, -A or-T) was performed using bbduk.sh. Human 
host reads were removed by mapping against the human genome (grch38) using the bbmap.sh utility. The quality metrics of the processed reads at each step were assessed using FastqC v0.11.9 (https://www.bioinformatics.babraham.ac.uk/projects/fastqc/).

Reads from different runs of the libraries prepared from the same genomic DNA sample were combined and used as an input for the assembly of the metagenome using metaSpades v3.14.0 $0^{38}$. Input reads were mapped back to the assembled contigs using bowtie2 (v.2.4.1) ${ }^{39}$. Binning of metagenomic contigs into metagenome assembled genomes (MAGs) of Mansonella and Wolbachia was performed using the BlobTools v1.1.1 software ${ }^{40}$ and additional curation. The bins annotated as "Proteobacteria" and "Nematoda" were analyzed further to retrieve sequences originating from Wolbachia. A cluster of "Proteobacteria" contigs in the blobplots of each of the metagenomes was analyzed further using blastn against $w$ Cle genome to verify their Wolbachia origin. These contigs were subsequently collected as respective Wolbachia assemblies from each of the isolates. The combined size of contigs collected from the Moz1 isolate was only $120 \mathrm{~kb}$, much smaller than a $1 \mathrm{Mb}$ genome typically seen for Wolbachia, and were not analyzed further. Similarly, in the Mpe 2 sample, only 12 contigs with a total size of $15 \mathrm{~kb}$ were identified as candidate Wolbachia sequence and were not analyzed further. The tight clusters of "Proteobacteria" contigs in the blobplots of Moz2 and Mpe1 were analyzed further as the wMoz and $w$ Mpe genome assemblies respectively. For the remaining clusters of metagenome contigs that were marked also "Proteobacteria" by BlobTools but had a distinctly different \%GC as compared to the Wolbachia contigs, blastn searches against the NCBI-nt database was performed to verify that they did not originate from the Wolbachia genome. All the bioinformatics programs were run using the default parameters unless otherwise stated. 


\section{Genome annotation and comparative analysis}

177 For both $w$ Mpe and $w$ Moz genomes, protein-coding genes, rRNA, tRNA, ncRNA genes and

178 pseudogenes were identified using the NCBI prokaryotic genome annotation pipeline ${ }^{41}$. Analysis

179 of sequence similarity and synteny between $w \mathrm{Moz}, w \mathrm{Mpe}$ and $w$ Cle genomes was carried out using the JupiterPlot tool (https://github.com/JustinChu/JupiterPlot) which uses minimap2 $2^{42}$ for whole-genome alignments. The parameters used in Jupiter plots are maxGap =100,000; minBundleSize $=1,000 ;$ m_ref_contig_cutoff $=500 ;$ gScaff $=500$. The parameter 'ng' was set to $100 \%$, so that all the contigs from the two assemblies being compared are displayed in the Jupiter plots. Whole-genome alignments of $w \mathrm{Moz}, w \mathrm{Mpe}$ as well as the $w$ Mhie genome against $w$ Cle as the reference genome were performed using the nucmer utility in MUMmer $4{ }^{43}$ with default parameters. The resulting alignment blocks were visualized as concentric Circos plots ${ }^{44}$ genomes was assessed using the BUSCO pipeline v5.0 beta using the "proteobacteria_odb10" reference dataset ${ }^{46}$. For global sequence comparisons across multiple Wolbachia genomes the average nucleotide identity (ANI) scores were calculated using the OrthoANIu tool v1.2 ${ }^{47}$. The pairwise ANI scores were used for hierarchical clustering of different Wolbachia, and a correlation plot was generated using $\mathrm{R}$ package corrplot v0.84

193 (https://github.com/taiyun/corrplot). For a more sensitive measure of sequence similarity and 194 divergence between closely related Wolbachia, digital DNA-DNA hybridization (dDDH) $195 \operatorname{scores}^{48}$ were computed using the recommended "formula 2" at the "Genome-to-Genome

197 protein coding genes across multiple Wolbachia genomes were inferred using OrthoFinder $198 \mathrm{v} 2.4^{49}$, and the results visually represented as UpSet plots ${ }^{50}$ using the R package UpSetR 
199

200

201

202

203

204

205

206

207

208

209

210

211

\section{Results}

213

214

215

216

217

218

219

220

v1.4.0 ${ }^{51}$. Insertion sequence (IS) elements were identified via the ISsaga web server ${ }^{52}$ and by parsing the annotations in GenBank format from NCBI-PGAP pipeline. Other transposons and Group II introns were also inferred via parsing the GenBank files. Phage-derived genes and regions were annotated by integrating the outputs from the PHASTER web server ${ }^{53}$, the PhiSpy v4.2.6 tool $^{54}$ (https://github.com/linsalrob/PhiSpy/), and manual curation based on GenBank annotations. Functional annotation of protein-coding genes was carried out using the eggNOGMapper ${ }^{55}$ web server (http://eggnogdb.embl.de/\#/app/emapper). The analysis of different metabolic pathways was based on the annotations of $w \mathrm{Cle}$ and $w \mathrm{Bm}$ genomes available in the KEGG database ${ }^{56}$. Pseudogene loci and the GenBank accession of the ortholog of their potential parent genes were annotated as a part of the NCBI-PGAP pipeline. If a pseudogene from multiple Wolbachia shared the same accession for the parent gene ortholog, it was considered to be a shared pseudogene.

\section{Metagenomic assembly and binning retrieves Wolbachia genomes from}

\section{complex samples}

All four isolates sequenced here were obtained as microfilariae from Mansonella-positive human subjects. The genomic DNA extracted from such clinical samples is unlikely to contain pure DNA originating from the parasite alone, instead each of the samples is expected to yield a complex assortment of mixed-organism DNA. The analysis of raw genome data from these samples therefore required a metagenomic assembly and binning approach to de-convolute parasite, symbiont and contaminants to permit species-specific genome assemblies. Further 
221 complicating correct assembly of Wolbachia genomes, is the presence of lateral gene transfer

222 events where symbiont DNA can integrate into the nuclear genome of the host. Appropriate steps

223 were taken to avoid including these HGT regions in the bacterial assemblies.

224

The assembled metagenome contigs were analyzed using BlobTools ${ }^{40}$, which is used to

225 identify clusters of contigs based on their GC content, and read coverage obtained by mapping

226 back input reads to the assembly, overlaid with sequence-identity based taxonomic classification.

227 Partitioning the data in this manner generated distinct blobs corresponding to the desired target

228 Wolbachia genomes from the mixed dataset, the genomes of the worm hosts and contaminating

229 microbiota. (Supplementary Figure S1). The Wolbachia contigs identified from the BlobTools

230 analysis were extracted as a separate bin of fasta sequences and analyzed further.

Of the two M. ozzardi isolates (Moz1 from Brazil and Moz2 from Venezuela), sufficient coverage was obtained from Moz2 that yielded a draft wMoz assembly of 1,073,310 bp in size comprised of 93 contigs. The N50 size of this assembly is $17.225 \mathrm{~kb}$ and the largest contig is N50 size of $10.041 \mathrm{~kb}$ and the largest contig is $28.485 \mathrm{~kb}$ (Table 1). 
To determine the nature of the gaps in the draft assemblies, the whole genome alignments were investigated in more detail. Interestingly, most of the regions absent from $w \mathrm{Moz}$ and $w \mathrm{Mpe}$ assemblies corresponded to regions containing IS element transposons in the $w$ Cle genome

246 (Figure 3). In a similar analysis using the recently available draft genome of $w$ Mhie, the missing

247 regions were again found to be associated with IS elements in $w$ Cle genome (Figure 3). Based on

248 this analysis, it appears that most of the protein coding regions have been assembled into these 249 draft genomes, and only the IS elements remain absent from the current assemblies. Due to their

250 repetitive nature, IS elements are known to present a technical hurdle in assembling complete 251 genomes, leading to fragmented assemblies ${ }^{57,58}$. $w \mathrm{Moz}$ and 864 protein coding genes in $w \mathrm{Mpe}$. A BUSCO analysis (v5.beta) was performed on

254 these proteins, to check for presence of the 219 genes conserved across most proteobacteria

255 ("proteobacteria_odb10" database in BUSCO). The BUSCO scores of $w \mathrm{Moz}$ and $w \mathrm{Mpe}$ were

$25677.6 \%$ and $74.4 \%$ respectively (Table 1), with such scores being typical for Wolbachia, even

257 with complete genomes. For comparison, the corresponding scores for other Wolbachia were

258 also calculated. The BUSCO scores for Wolbachia $w \mathrm{Ov}$ from the human filarial parasite

259 Onchocerca volvulus and wOo, present in the related bovine parasite Onchocerca ochengi are

$26076.7 \%$ and $75.8 \%$ respectively, and $79.5 \%$ for $w \mathrm{Bm}$ from the human filarial parasite Brugia

261 malayi (Supplementary Figure S2). Within supergroup F, the BUSCO score for filarial

262 Wolbachia $w$ Mhie is $79 \%$ and is $81.7 \%$ for the arthropod Wolbachia $w$ Cle. 
The metagenomic assembly and binning approach also enables the measurement of the relative amounts of Wolbachia in different isolates, by comparing the sequencing reads coverage

267 of the Mansonella and Wolbachia specific contig bins in the metagenomic assemblies (Table 2).

268 In the M. ozzardi sample (Moz2), the median coverage of contigs classified as Mansonella was

269 observed to be 360X, while that of the Wolbachia contigs was found to be 13X. Assuming 1000

270 nematode cells per microfilaria ${ }^{59}$, each parasite is estimated to harbor 37 Wolbachia cells. For the

271 other M. ozzardi isolate (Moz1) the median coverage for nematode contigs was only 57X and

272 this low coverage is the most likely reason why this isolate did not yield sufficient Wolbachia

273 contigs in its metagenomic assembly. Therefore, the titer calculations were not performed for the

274 Moz1 isolate as they would not be robust at such low coverage and incomplete assembly. For the

275 M. perstans sample (Mpe1), the median coverage of nematode contigs was $1032 \mathrm{X}$ and the

276 Wolbachia coverage was 30X, yielding an estimated titer of 30 Wolbachia per microfilaria. In

277 the other M. perstans isolate Mpe2, where only 12 Wolbachia contigs were produced in the

278 metagenomic assembly, the median coverage for nematode contigs is relatively high $633 \mathrm{X}$,

279 indicating that the lack of Wolbachia contigs is not due to insufficient depth of coverage and is

280 rather most likely due to a very low titer of Wolbachia in this sample. Since both isolates were

281 collected in Cameroon, these observations point to variation in Wolbachia titers within the same

282 species in a geographical area and could explain the conflicting reports on detection of

283 Wolbachia in M. perstans ${ }^{25-27,31-33}$. 
The sequence similarity and divergence between different Wolbachia genomes from filarial nematodes, a plant parasitic nematode, and arthropod hosts representing different supergroups (Supplementary Table S1) was measured using the genome-wide average nucleotide identity (gANI) and digital DNA-DNA hybridization (dDDH) scores. The gANI scores (Figure 4A) for closely related sympatric Wolbachia are: $99 \%$ for the wOo : wOv pair from Onchocerca spp. and $99 \%$ for the $w \mathrm{Bm}: w \mathrm{Bpa}$ pair from Brugia spp. Within supergroup $\mathrm{F}$, high gANI scores were observed as expected; namely $97 \%$ between the $w \mathrm{Moz}: w \mathrm{Mpe}$ pair, and $92 \%$ between $w \mathrm{Moz}$ : $w$ Cle and $w$ Mpe : $w$ Cle pairs (Figure 4A). $w$ Mhie shared a higher similarity with $w$ Cle (95\%), than to $w \mathrm{Moz}$ and $w \mathrm{Mpe}(92 \%)$. Comparisons between Clade F members and those of other clades generated gANI scores of a maximum value of $85 \%$ (Figure $4 \mathrm{~A}$ ).

Using $\mathrm{dDDH}$, a more sensitive metric for sequence divergence between very closely related species, the $\mathrm{dDDH}$ score of the $w \mathrm{Moz}: w \mathrm{Mpe}$ pair was found to be $71.7 \%$ (Figure 4B). For comparison, the dDDH scores for other pairs of closely related Wolbachia are: $96.1 \%$ for the wOo : wOv pair from Onchocerca spp. (Figure 4C) and 93.3\% for the $w \mathrm{Bm}:$ w Bpa pair from Brugia spp. (Figure 4D). Within supergroup F, the dDDH scores of more diverged pairs, namely $w \mathrm{Moz}: w \mathrm{Cle}$ and $w \mathrm{Mpe}: w \mathrm{Cle}$ are only $\sim 44 \%$, while the scores obtained for $w$ Mhie : $w$ Cle is considerably higher at $61.8 \%$.

\section{The core proteome of filarial $w \mathrm{Mpe}$ and $w \mathrm{Moz}$}

Orthologs for $w \mathrm{Moz}$ and $w$ Mpe proteins were identified with OrthoFinder ${ }^{49}$ using the predicted proteomes of various Wolbachia, including complete genomes representing each arthropod Wolbachia supergroup, all filarial Wolbachia genomes and the only Wolbachia genome from a 
plant parasitic nematode (Supplementary Table 2). From a total input set of 17,720 proteins, $96 \%$ of proteins $(n=17,020)$ could be clustered into 1,440 orthogroups that were present in at least two Wolbachia proteomes, while $1.5 \%$ of proteins $(n=253)$ were grouped into 65 orthogroups that were species-specific (Supplementary Table S2-A). Only 2.5\% $(\mathrm{n}=447)$ of all the input proteins could not be assigned to any orthogroups. For $w \mathrm{Moz}$ and $w \mathrm{Mpe}, 851$ and 821 of their protein-coding genes ( $95 \%)$ were assigned to one of the 1,440 shared orthogroups, while 35 of $w \mathrm{Moz}$ proteins and 39 of $w \mathrm{Mpe}$ proteins (less than $5 \%$ in both cases) could not be assigned to any orthogroup (Supplementary Table S2-B, C). These trends are very similar for the other genomes analyzed, with more than $95 \%$ of proteins for each Wolbachia assigned to a shared orthogroup, and the proportion of potentially genome-specific orthogroups being lower than 5\% (Supplementary Table S2-C). The only exception to this trend was observed for the Folsomia candida Wolbachia ( $w$ Fol) genome, for which $9.6 \%$ of its protein-coding genes were clustered into $w$ Fol-specific orthogroups, while $9 \%$ of its proteins could not be assigned to any orthogroups.

A total of 411 orthogroups were found to be conserved across all the Wolbachia analyzed (Figure 5). Interestingly, 29 orthogroups were found that are present in $w \mathrm{Mpe}$ and $w \mathrm{Moz}$, but not in the two other clade F Wolbachia, wCle and wMhie. However, further analysis revealed most of these genes to be hypothetical proteins with a median size of 80 aa. From these patterns of shared orthologs, it can be seen that the set of proteins encoded in supergroup F Wolbachia genomes, particularly $w \mathrm{Moz}$ and $w \mathrm{Mpe}$, do not deviate significantly from those of other filarial Wolbachia. 


\section{Mobile genetic elements in $w \mathrm{Moz}$ and $w$ Mpe genomes}

331

332

333

334

335

336

337

338

339

340

341

342

343

344

Genes encoding various kinds of transposable elements, including IS elements, were identified based on annotation descriptions obtained from the PGAP pipeline. The $w$ Moz genome assembly has 25 transposable elements (3 intact protein coding genes and 22 pseudogenes, Supplementary Table 3A), while the $w$ Mpe genome assembly contains 23 transposable elements ( 1 intact protein coding gene, 22 pseudogenes, Supplementary Table 3B). Applying the same search strategy to the $w$ Mhie genome assembly identified 66 transposon elements (16 intact protein coding genes, 50 pseudogenes, Supplementary Table 3C). Within the set of these transposable elements, the $w$ Moz assembly harbors only 1 intact IS3 family gene and 12 pseudogenes from various families of IS elements. The $w$ Mpe draft genome contains 10 pseudogenes from various IS families but no intact IS element gene, while 14 intact and 42 pseudogenized IS elements were identified in the wMhie genome. Additional search for IS elements in $w \mathrm{Moz}$ and $w \mathrm{Mpe}$ genome assemblies using the ISsaga web-server ${ }^{52}$ did not reveal any other IS elements in these genomes. In the complete $w$ Cle genome, ISsaga web-service could identify 149 IS elements, with the IS5 family being the most abundant (121 copies). The low number of IS elements observed in $w \mathrm{Moz}$ and $w \mathrm{Mpe}$ is most likely due to the limitations of short-read data in capturing repeated elements.

Group II introns are a common mobile element found in high copy numbers in many Wolbachia genomes, e.g. 53 copies in the $w$ AlbB genome ${ }^{57}$. Both the $w \mathrm{Moz}$ and $w \mathrm{Mpe}$ assembly contain only 1 pseudogene derived from a Group II intron, while no intact gene or pseudogene for these elements could be found in $w$ Mhie and $w$ Cle genomes as reported earlier ${ }^{6}$.

A search for prophage elements identified 9 phage derived genes in both $w \mathrm{Moz}$ and $w$ Mpe assemblies of which 4 have been pseudogenized in both Wolbachia (Supplementary Table 
3D). A gene encoding gp6-like head-tail connector phage protein and a pseudogene derived from

353 a phage portal protein were found only in $w \mathrm{Moz}$, while a pseudogene derived from major tail-

354 tube phage protein was found only in $w \mathrm{Mpe}$. This is in accordance with the trend of none or very

355 few phage derived genes in filarial Wolbachia genomes ${ }^{6}$.

\section{Analysis of various biological pathways in $w \mathrm{Moz}$ and $w \mathrm{Mpe}$ genomes}

For a detailed functional analysis, the proteins encoded by the $w \mathrm{Moz}$ and $w \mathrm{Mpe}$ genomes were annotated using the eggNOG database (Supplementary Tables S4A-B). Additionally, biosynthetic pathway annotations for the $w$ Cle genome were obtained from the KEGG database and corresponding orthologs were identified in $w \mathrm{Moz}$ and $w \mathrm{Mpe}$. The genes encoding members of various metabolic pathways characteristic of Wolbachia proteomes, namely the heme pathway, purine and pyrimidine biosynthesis pathways, riboflavin metabolism, and Type IV secretion systems were found to be mostly present in $w \mathrm{Moz}$ and $w \mathrm{Mpe}$ (Supplementary Tables S5A-E). Ribosomal protein subunits, a common target for anti-Wolbachia drugs such as

367 were also present in both $w \mathrm{Moz}$ and $w \mathrm{Mpe}$.

\section{Biotin biosynthesis genes are absent in $w$ Mpe and $w$ Moz draft assemblies}

370 The bedbug Wolbachia wCle genome harbors an operon encoding the enzymes involved in

371 biosynthesis of biotin, supporting a nutritional mutualism between the bedbug and its

$372 W_{\text {olbachia }}^{29}$. Interestingly, this operon has most likely been laterally acquired by $w$ Cle from a 
373 co-infecting Rickettsia or Cardinium symbiont ${ }^{29}$. Given the paucity of genome data on other

374 members of supergroup F, it is not clear whether this pathway is also present in other Wolbachia

375 from this supergroup. Searches of the $w \mathrm{Moz}$ and $w \mathrm{Mpe}$ proteomes did not reveal any members

376 of the biotin pathway suggesting that unlike the $w$ Cle and bedbug symbiosis, biotin

377 supplementation is not the basis of mutualism between these Wolbachia and their filarial host.

378 The absence of biotin pathway genes is unlikely to be due to the incomplete nature of the draft

379 assemblies. Given the high BUSCO score ( $80 \%)$ of the assemblies, there is a high probability

380 that at least fragments of a few of the 6 genes in the biotin pathway would have been detected if

381 present in the genomes. Further, the biotin pathway genes were also found to be absent in the

382 draft genome of $w$ Mhie - the other filarial Wolbachia from the supergroup $\mathrm{F}^{6}$. In contrast, the

383 biosynthetic genes of other vitamin pathways present in $w$ Cle were easily found in $w$ Mpe and

$384 w$ Moz (Supplementary Table S5A-E).

Shared pseudogenes point to a common ancestor of $w \mathrm{Moz}$ and $w \mathrm{Mpe}$ being

present in the common ancestor of $M$. perstans and $M$. ozzardi

Intracellular bacteria such as Wolbachia are expected to accumulate deleterious mutations over

evolutionary timescales, with one consequence being the formation of pseudogenes ${ }^{61}$. Analysis

390 of these pseudogenes can also shed light on the nature of genes that are dispensable during a

391 symbiotic association with the host organism. Pseudogenes in $w \mathrm{Moz}(\mathrm{n}=118)$ and $w \operatorname{Mpe}(\mathrm{n}=$

392 132) were annotated as a part of the NCBI PGAP pipeline and compared with corresponding

393 annotations in $w$ Mhie $(\mathrm{n}=87)$ and $w$ Cle $(\mathrm{n}=212)$. These annotations include information on the

394 ortholog of ancestral protein-coding gene for each pseudogene (Supplementary Table 6). If the 
same ancestral gene was found to have converted to a pseudogene in two or more Wolbachia, it was counted as a shared pseudogene (Figure 6). Transposons such as IS elements were excluded from this analysis due to their tendency to frequently pseudogenize.

For each Wolbachia, the majority of its pseudogenes were found to be unique to its genome, and only 1 pseudogene was shared among all the 4 Wolbachia. In pairwise comparisons of Wolbachia, only 2 to 7 pseudogenes were common among pairs of Wolbachia, except for the $w$ Moz : $w$ Mpe pair, which share a much larger set $(\mathrm{n}=38)$. This large proportion of common pseudogenes suggests that these pseudogenes were already present in the last common ancestor (LCA) of $w \mathrm{Moz}$ and $w \mathrm{Mpe}$.

\section{Discussion}

To date, there are only two complete Wolbachia genomes available from human filarial parasites; $w \mathrm{Bm}$ from Brugia malayi ${ }^{62}$, one of several parasites responsible for lymphatic filariasis or elephantiasis, and $w \mathrm{Ov}$ from $O$. volvulus, the causative agent of onchocerciasis or river blindness ${ }^{63}$. These genomes belong to different phylogenetic lineages $-w \mathrm{Bm}$ is a member of supergroup $\mathrm{D}$, whereas $w \mathrm{Ov}$ has been assigned to supergroup $\mathrm{C}$. This is the first report describing the genomes of supergroup F Wolbachia from Mansonella. In this study a metagenomic approach was utilized to successfully retrieve the $w$ Moz genome from one isolate of M. ozzardi collected in Venezuela and the $w$ Mpe genome from one isolate of $M$. perstans from Cameroon. The metagenomic assembly and binning approach that combines information from GC content of assembled contigs in conjunction with their read-coverage played a critical role in the generation of high-quality, draft genomes for $w \mathrm{Mpe}$ and $w \mathrm{Moz}$, from complex clinical 
417 samples containing a mixture of DNA from the human host, the filarial parasite and its

418 intracellular symbiont and possibly other organisms that might be present in humans. The use of

419 BlobTools ${ }^{40}$ for binning of contigs has been successfully employed previously to disentangle

420 genomes of the Wolbachia wDimm from its host Dirofilaria immitis ${ }^{64,65}$, a filarial parasite of

421 dogs and cats that occasionally infects humans. endosymbiont present in the worm host. In a previous analysis of Wolbachia burden in different

424 strains and stages of $D$. immitis, use of a BlobTools-like approach identified distinct blobs of contigs with levels of coverage consistent with high or low Wolbachia abundance ${ }^{65}$. Performing

426 a similar analysis for Mansonella samples in the current study, the number of Wolbachia was

427 estimated to be around 30 cells per microfilaria for samples Moz2 and Mpe1. This level of

428 Wolbachia in Mansonella is considerably lower than the reported levels of 100 Wolbachia per microfilaria in Brugia malayi ${ }^{66}$. For the other M. perstans sample Mpe2 obtained from the same geographical location as Mpe1, only 12 small contigs corresponding to Wolbachia were found at very low coverage, demonstrating that the endosymbiont was present at extremely low levels, 432 precluding accurate titer estimation. These observations indicate that even within a locality, 433 Wolbachia levels can vary between different isolates, and could be a possible explanation for 434 conflicting reports on the presence and absence of Wolbachia in M. perstans ${ }^{25-27,31-33}$. In addition 435 to potential natural variation, previous exposure to antibiotics can also be a cause of very low 436 Wolbachia levels, and could be another explanation for the occasional failures to detect 437 Wolbachia in PCR-based assays ${ }^{25-27,31-33}$. 
440

441

442

443

444

445

446

447

448

449

450

451

452

453

454

455

456

457

458

459

460

461

462

perstans microfilaremia supports an essential role of Wolbachia in this species ${ }^{25-27}$. Interestingly, Wolbachia has recently been reported in Mansonella sp. "DEUX" 33, a potentially new species of Mansonella parasite of humans, which is closely related to M. perstans ${ }^{67}$. To date, Wolbachia has been consistently found in all $M$. ozzardi isolates ${ }^{5,28}$, including the two isolates in the current study. Therefore, Wolbachia is most likely essential and present in all Mansonella parasites of humans, but can be present at different levels, sometimes below the limit of detection in $M$. perstans.

Analysis of whole-genome alignments of the $w \mathrm{Mpe}$ and $w \mathrm{Moz}$ to the complete $w \mathrm{Cle}$ genome in the same supergroup F identified regions of high sequence similarity and synteny, and also revealed that the location of alignment gaps mainly corresponded to the locations of IS elements in $w$ Cle genome. Due to their repetitive nature, the high-copy number IS elements are challenging to assemble with short-read data alone, and produce assemblies fragmented into multiple contigs, with the contig breakpoints mostly caused by the un-assembled IS elements ${ }^{57,58}$. Based on these observations, it is quite likely that both $w \mathrm{Moz}$ and $w \mathrm{Mpe}$ contain some as yet unidentified IS elements present in high copy numbers in their genomes. Consistent with this, 10 pseudogenized IS elements could be identified in both $w \mathrm{Moz}$ and $w \mathrm{Mpe}$ assemblies and only one 1 intact IS element could be identified in $w \mathrm{Moz}$. The pseudogene copies diverge in sequence from their parent gene and therefore become low-copy variants that can be successfully assembled even with short-read data. Assuming at least one IS element in each of the alignment gaps, the number of IS elements in $w \mathrm{Moz}$ and $w \mathrm{Mpe}$ is predicted to be similar to the number of their contigs, that is around 100 copies. Using the same annotation pipeline on the $w$ Mhie draft genome, 42 pseudogenized and 14 intact IS elements could be identified. Together, these observations point to presence of around 50 to 100 IS elements in filarial Wolbachia from 
supergroup F. This is in contrast to the trend in supergroups $\mathrm{C}$ and $\mathrm{D}$, where fewer than 5 IS elements could be identified in complete genomes ${ }^{6,57,68}$. It is common for arthropod Wolbachia from other supergroups (such as A and B) to have hundreds of copies of IS elements, for example, comprising as high as $13 \%$ of the genome sequence in $w \mathrm{AlbB}^{57}$. Supergroup $\mathrm{F}$ is unique in having members from both filarial and arthropod hosts. It is interesting that the number of IS elements in filarial Wolbachia from this clade is intermediate between those observed in other arthropod and filarial Wolbachia.

The $w$ Moz and $w$ Mpe genomes enable analysis of their evolutionary history from multiple perspectives. Insights can be gained from comparison of pseudogene content across different supergroup F Wolbachia. Since pseudogenes are evolutionary accidents, and the probability of such disruptions happening in the same gene in two independent lineages is low, the presence of multiple shared pseudogenes suggests that these pseudogenes were already present in the last common ancestor (LCA) of $w \mathrm{Moz}$ and $w \mathrm{Mpe}$. Since these endosymbionts are restricted to survive only within their host nematodes, it can be extrapolated that the LCA of $w \mathrm{Moz}$ and $w \mathrm{Mpe}$ should have been already present in the last common ancestor of $M$. ozzardi and M. perstans. After the split, both $w \mathrm{Moz}$ and $w \mathrm{Mpe}$ have continued to accumulate lineagespecific pseudogenes $(\mathrm{n}=56$ for $w \mathrm{Moz}, \mathrm{n}=62$ for $w \mathrm{Mpe})$.

Comparisons of genome-wide sequence similarity within supergroup $\mathrm{F}$ were also made using the dDDH score. The dDDH scores simulate the results of DNA : DNA hybridization, and provide a sensitive measure of sequence similarity and divergence between closely related bacteria at the whole-genome level. Additionally, this measure has been used to determine species boundaries, with dDDH scores above $70 \%$ typically indicating the same species ${ }^{48}$. The $\mathrm{dDDH}$ score for the $w \mathrm{Moz}: w \mathrm{Mpe}$ pair was found to be $71.7 \%$, while the corresponding scores 
between other closely related but distinct Wolbachia e.g., $w \mathrm{Bm}: w \mathrm{Bpa}$ pair and $w \mathrm{Oo}: w \mathrm{Ov}$ pair were even higher (93.3\% and $96.1 \%$ respectively). Since each of these Wolbachia pairs (e.g., $w \mathrm{Moz}$ and $w \mathrm{Mpe})$ are found in distinct nematode hosts and cannot exchange genetic material, they are biologically distinct species. Therefore, the threshold used to delineate species boundaries in free-living bacteria is most likely not directly applicable to intracellular endosymbionts, which would have very different population dynamics as compared to free-living species. The dDDH score is nonetheless a useful metric for measuring sequence divergence. The lower $\mathrm{dDDH}$ scores of the $w \mathrm{Moz}: w \mathrm{Mpe}$ pair as compared to the $w \mathrm{Bm}: w \mathrm{Bpa}$ and $w \mathrm{Ov}: w \mathrm{Oo}$ pairs indicates that the nucleotide sequence divergence between Wolbachia of Mansonella is much higher than the corresponding divergence in Wolbachia from either the Brugia or Onchocerca pair. While $w \mathrm{Moz}$ and $w$ Mpe are both derived from filarial parasites of humans, they still show higher divergence than other pairs that comprise of one Wolbachia from a human filarial parasite $(w \mathrm{Bm}, w \mathrm{Ov})$ and one from a non-human filaria $(w \mathrm{Bpa}, w \mathrm{Oo})$. One potential cause for the higher divergence observed in $w \mathrm{Moz}: w \mathrm{Mpe}$ pair could be the Mansonella lineages splitting into two species (M. perstans and M. ozzardi) much earlier than the splitting within Brugia or Onchocerca lineages. Additionally, other ecological factors such as geographical location and vectors involved might contribute to sequence divergence of filarial nematodes and their endosymbionts.

Orthology analysis and annotation of key biological pathways were performed to infer biological functions of the $w \mathrm{Moz}$ and $w \mathrm{Mpe}$ endosymbionts. These comparisons included Wolbachia genomes from other filarial hosts, as well as representative genomes from other supergroups. Similar to other Wolbachia, genes encoding enzymes of various biosynthetic pathways, namely heme, purine, pyrimidine and riboflavin were present in $w \mathrm{Moz}$ and $w \mathrm{Mpe}$. 
509 The proposed anti-Wolbachia drug target PPDK involved in the glycolysis/gluconeogenesis

510 pathway ${ }^{60}$ was conserved in all Wolbachia. Doxycycline, a commonly used anti-Wolbachia

511 antibiotic that targets protein synthesis machinery, is reported to be effective against Mansonella

512 perstans ${ }^{25-27}$. Consistent with this, all ribosomal subunits were found in $w \mathrm{Moz}$ and $w \mathrm{Mpe}$.

The supergroup F Wolbachia wCle provides biotin as a nutritional supplement to its host

514 bedbug and contains the complete pathway for biotin biosynthesis ${ }^{29}$. However, none of the genes

515 of the biotin pathway could be found in $w \mathrm{Moz}, w \mathrm{Mpe}$ or in $w$ Mhie, indicating that biotin

516 supplementation is not the basis of their filarial symbiosis. The biotin pathway in the $w$ Cle

517 genome has been acquired via LGT from another Rickettsia ${ }^{29}$ and does not seem to be a feature

518 common to other Wolbachia of supergroup F.

In summary, high quality draft genomes of Wolbachia wMoz and $w \mathrm{Mpe}$ from human filarial parasites $M$. ozzardi and $M$. perstans have been generated from genomic analysis of

521 multiple isolates from different continents. These genomes provide an important resource for

522 studies of symbiosis, evolution, comparative genomics, as well as searches for new drug targets.

523 In addition, the successful use of a metagenomic assembly and binning approach for obtaining

524 endosymbiont genomes exemplifies the wider applicability of this method to other systems

525 where the DNA samples can only be obtained as complex mixtures of multiple organisms. Since

526 the genomes from the host and endosymbiont are sequenced simultaneously, this approach

527 uniquely enables a method for directly and robustly estimating the relative abundance of 528 different organisms in a metagenomic sample. 
531 The authors thank Jeremy Foster for helpful discussions and comments on the manuscript, and

532 Laurie Mazzola and Danielle Fuchs from the DNA sequencing core at New England Biolabs.

533 The work was inspired by Don Comb and funded by New England Biolabs. Field research in

534 Brazil was supported by the Fundação de Amparo à Pesquisa do Estado de São Paulo (FAPESP),

535 research grant to M.U.F. (2013/12723-7) and doctoral scholarship to N.F.L. (2013/ 26928-0)

536

537 Author Contributions

538 N.F. L., M. U. F., F.F. F., S.W. collected the clinical samples. A.S., Z. L, C.B. P., L. E.

539 performed the sequencing experiments and bioinformatics analysis. A.S., Z. L, C.B. P., L.E.,

540 C.K.S.C. analyzed the data. A.S., Z. L, C.B. P., C.K.S.C wrote the initial manuscript. A.S., Z. L,

541 C.B. P., L.E., M. U. F., S.W., C.K.S.C and all authors contributed to the final manuscript. 


\section{Tables}

545 Table 1. Characteristics of genomes of wMoz and wMpe, and other Wolbachia from

\begin{tabular}{|r|c|c|c|c|}
\hline & $w \mathbf{M o z}$ & $\boldsymbol{w M p e}$ & $\boldsymbol{w}$ Mhie & $\boldsymbol{w C l e}$ \\
\hline Assembled genome size & $1,073,310$ & $1,058,123$ & $1,025,329$ & $1,250,060$ \\
\hline Number of contigs & 93 & 170 & 208 & 1 \\
\hline Contig N50 size & $17.225 \mathrm{~kb}$ & $10.041 \mathrm{~kb}$ & $6.845 \mathrm{~kb}$ & - \\
\hline \%redicted genes & 1,079 & 1,058 & 1,081 & 1,238 \\
\hline Predicted proteins & 838 & 864 & 907 & 1,020 \\
\hline Predicted pseudogenes & 145 & 153 & 137 & 177 \\
\hline tRNA genes & 36 & 34 & 30 & 34 \\
\hline BUSCO score (\%) & 78.1 & 75.3 & 79.0 & 81.7 \\
\hline & & & & \\
\hline
\end{tabular}

Table 2. Estimation of Wolbachia levels per microfilaria

\begin{tabular}{|c|l|l|l|l|l|l|}
\hline & & $\begin{array}{l}\text { Median } \\
\text { coverage of } \\
\text { Mansonella } \\
\text { contigs (M) }\end{array}$ & $\begin{array}{l}\text { Number and } \\
\text { total size of } \\
\text { assembled } \\
\text { Wolbachia } \\
\text { contigs } \\
\text { name, } \\
\text { Location }\end{array}$ & $\begin{array}{l}\text { Wolbachia } \\
\text { genome } \\
\text { assembled }\end{array}$ & $\begin{array}{l}\text { Median } \\
\text { coverage of } \\
\text { Wolbachia } \\
\text { contigs (W) }\end{array}$ & $\begin{array}{l}\text { Number of } \\
\text { Wolbachia } \\
\text { per } \\
\text { microfilaria* } \\
\text { (1000 x } \\
\text { W/M) }\end{array}$ \\
\hline M. ozzardi & Moz1, Brazil & 57 & $\begin{array}{c}173 \text { contigs, } \\
0.12 \mathrm{Mb}\end{array}$ & No & 3 & $\mathrm{NA}^{\#}$ \\
\hline M. ozzardi & $\begin{array}{c}\text { Moz2, } \\
\text { Venezuela }\end{array}$ & 360 & $\begin{array}{c}93 \text { contigs, } \\
1.073 \mathrm{Mb}\end{array}$ & $w \mathrm{Moz}$ & 13 & 37 \\
\hline
\end{tabular}


549

550

551

552

$553 \quad$ Figure Legends

\begin{tabular}{|c|c|c|c|c|c|c|}
\hline M. perstans & $\begin{array}{c}\text { Mpe1, } \\
\text { Cameroon }\end{array}$ & 1,032 & $\begin{array}{c}170 \text { contigs, } \\
1.058 \mathrm{Mb}\end{array}$ & $w \mathrm{Mpe}$ & 30 & 30 \\
\hline M. perstans & $\begin{array}{c}\text { Mpe2, } \\
\text { Cameroon }\end{array}$ & 633 & $\begin{array}{c}5 \text { contigs, } \\
8.781 \mathrm{~kb}\end{array}$ & No & NA $^{\#}$ & NA $^{\#}$ \\
\hline
\end{tabular}

* Assuming 1000 cells per microfilaria

\# Not calculated due to the very low number or coverage of Wolbachia contigs

554

Figure 1. Jupiter plot showing high synteny between the de novo assembled $w$ Moz and

555 $w$ Mpe genomes

556

The Wolbachia genomes assembled from two biologically and geographically distinct isolates

557 display a high level of synteny and inter-genome consistency. The genomes were aligned using

558 minimap2 and visualized using the JupiterPlot software. The $w$ Moz contigs are displayed as

559 colored boxes in the left semi-circle, while the $w$ Mpe contigs are shown as gray boxes in the

560 right semi-circle. The syntenic regions are marked by bands connecting contigs from one

561 assembly to the corresponding region in the other assembly. Regions of rearrangement are shown

562 as bands crisscrossing the horizontal bands of the syntenic regions.

564 Figure 2. Jupiter plots showing synteny between (A) $w \mathrm{Moz}$ and (B) $w \mathrm{Mpe}$ with the $w \mathrm{Cle}$ 
The $w$ Cle chromosome is represented in linear form as the blue semi-circle on the left in both

567

568

569

570

571

572

573

574

575

576

577

578

579

580

581

582

583

584 panels. The gray boxes represent contigs of (A) $w \mathrm{Moz}$ draft genome, and (B) $w \mathrm{Mpe}$ draft

genome. The horizontal bands connect the conserved regions across the two Wolbachia. Regions

of re-arrangements are shown as bands crisscrossing the horizontal bands of syntenic regions.

Figure 3. Comparison of genome sequences of all supergroup F Wolbachia

Whole genome alignments of $w \mathrm{Moz}$ (blue ring), $w \mathrm{Mpe}$ (light-blue ring) and $w$ Mhie (innermost gray ring) to the $w$ Cle chromosome (outermost black circle) are visualized as a circos plot. The red bars mark the locations of the IS elements in the $w$ Cle genome.

Figure 4. Global comparisons between multiple Wolbachia genomes using genome-wide Average Nucleotide Identity (gANI) scores, and digital DNA:DNA Hybridization (dDDH) scores.

(A) gANI scores were calculated between pairs of Wolbachia from filarial and plant-parasitic nematodes, and the arthropod Wolbachia wCle. Hierarchical clustering of pairwise gANI scores, presented as a correlation matrix, ordered the various Wolbachia in a pattern recapitulating their supergroup assignments. The red lines mark the boundaries between clusters representing different supergroups. (B) dDDH scores between members of supergroup F (C) dDDH scores between members of supergroup C, and (D) dDDH scores between members of supergroup D. 
Figure 5. Orthogroups conserved in $w$ Mpe and $w$ Moz compared to 18 other Wolbachia genomes

588

589

590

591

592

593

594

595

596

597

598

599

600

601

602

603

604

Orthology relationships between proteins encoded by wMoz and wMpe and 18 other Wolbachia were determined using OrthoFinder and visualized as UpSet plots. Black dots represent presence of an orthogroup while light gray dots denote absence. The Wolbachia supergroup is indicated except for the currently un-assigned Wolbachia from Ctenocephalides felis $\mathrm{J}$ (wCfeJ) and Ctenocephalides felis $\mathrm{T}(w \mathrm{CfeT})$.

Figure 6. Venn diagram comparing pseudogenes in supergroup F Wolbachia

Pseudogenes for $w \mathrm{Moz}$ and $w \mathrm{Mpe}$ were annotated as a part of the NCBI PGAP pipeline.

Transposons such as IS elements were excluded from this analysis due to their tendency to frequently convert into pseudogenes.

\section{Supplementary Information}

Figure S1. BlobTools based binning of the metagenomes assembled from Mansonella isolates (A) M. ozzardi isolate Moz1, from Brazil (B) M. ozzardi isolate Moz2, from

Venezuela (C) M. perstans isolate Mpe1, from Cameroon and (D) M. perstans isolate Mpe2, from Cameroon. 
605 Each dot in the plot represents a contig in the assembled metagenome, plotted by its \%GC

606 content on the $\mathrm{x}$-axis and $\log 10$ of its coverage on the $\mathrm{y}$-axis. The dot size is scaled according to

$607 \log 10$ of the length of each contig. Colors were assigned based on taxonomic annotations from

608 BlobTools and manual annotations. Bins that were finally assigned to the Mansonella host and

609 the Wolbachia endosymbiont are marked by enclosing boxes. Boxes with dashed borders

610 indicate locations of expected Wolbachia contigs, but very few or very small contigs were

611 actually recovered for these samples.

612 Figure S2. BUSCO scores of various Wolbachia

613 The BUSCO pipeline was used to measure the proportion of highly conserved, single copy

614 orthologs (BUSCO groups). The set of reference BUSCO groups was set to the lineage

615 “proteobacteria_odb10”, which contains 219 BUSCOs derived from proteobacterial species. The

$616 w$ Oo genome has the lowest BUSCO score, marked by a vertical dotted line. The supergroups

617 are indicated within a parenthesis after the corresponding Wolbachia names.

618 Table S1. Wolbachia genomes used for comparative analysis with $w \mathrm{Moz}$ and $w \mathrm{Mpe}$

619 genomes

620 Table S2: Orthology analysis using OrthoFinder

621 Table S2-A. Orthogroups and their member genes for different Wolbachia

622 Table S2-B. Genes not assigned to any orthogroups.

623 Table S2-C. Overall OrthoFinder statistics for each Wolbachia genome analyzed. 
624 Table S3: Mobile genetic elements in $w$ Moz and $w$ Mpe genome assemblies. (A) Transposons

625 and Group II introns in wMoz (B) Transposons and Group II introns in $w$ Mpe (C) Transposons in

$626 w$ Mhie (D) Phage derived genes in $w \mathrm{Moz}$ and $w \mathrm{Mpe}$

627 Table S4: eggNOG annotations of (A) wMoz and (B) wMpe proteins

628 Table S5: Various biological pathways in $w \mathrm{Moz}$ and $w \mathrm{Mpe}$ and other supergroup F

629 Wolbachia. (A) Heme pathway (B) Purine (C) Pyrimidine (D) Type IV and Type VI secretion

630 systems

631 Table S6: Pseudogenes present in the genomes of supergroup F Wolbachia 
633

634

635

636

637

638

639

640

641

642

643

644

645

646

647

648

649

650

651

652

653

654

655

\section{References}

1. Werren, J. H., Baldo, L. \& Clark, M. E. Wolbachia: master manipulators of invertebrate biology. Nat. Rev. Microbiol. 6, 741-751 (2008).

2. Zug, R. \& Hammerstein, P. Bad guys turned nice? A critical assessment of Wolbachia mutualisms in arthropod hosts. Biol. Rev. 90, 89-111 (2015).

3. Taylor, M. J., Bandi, C. \& Hoerauf, A. Wolbachia Bacterial Endosymbionts of Filarial Nematodes. in Advances in Parasitology (eds. Baker, J. R., Muller, R. \& Rollinson, D.) vol. 60 245-284 (Academic Press, 2005).

4. Pfarr, K. M. \& Hoerauf, A. M. Antibiotics which target the Wolbachia endosymbionts of filarial parasites: a new strategy for control of filariasis and amelioration of pathology. Mini Rev. Med. Chem. 6, 203-210 (2006).

5. Lefoulon, E. et al. Breakdown of coevolution between symbiotic bacteria Wolbachia and their filarial hosts. PeerJ 4, e1840 (2016).

6. Lefoulon, E. et al. Diminutive, degraded but dissimilar: Wolbachia genomes from filarial nematodes do not conform to a single paradigm. Microb. Genomics 6, e000487 (2020).

7. Lefoulon, E. et al. Pseudoscorpion Wolbachia symbionts: diversity and evidence for a new supergroup S. BMC Microbiol. 20, 188 (2020).

8. Downes, B. \& Jacobsen, K. A systematic review of the epidemiology of mansonelliasis. Afr. J. Infect. Dis. 4, (2010).

9. Simonsen, P. E., Onapa, A. W. \& Asio, S. M. Mansonella perstans filariasis in Africa. Acta Trop. 120, S109-S120 (2011).

10. Lima, N. F., Aybar, C. A. V., Juri, M. J. D. \& Ferreira, M. U. Mansonella ozzardi: a neglected New World filarial nematode. Pathog. Glob. Health 110, 97-107 (2016). 
11. Mediannikov, O. \& Ranque, S. Mansonellosis, the most neglected human filariasis. New Microbes New Infect. 26, S19-S22 (2018).

12. Ta-Tang, T.-H., Crainey, J. L., Post, R. J., Luz, S. L. \& Rubio, J. M. Mansonellosis: current perspectives. Res. Rep. Trop. Med. 9, 9-24 (2018).

13. Bélard, S. \& Gehringer, C. High Prevalence of Mansonella Species and Parasitic Coinfections in Gabon Calls for an End to the Neglect of Mansonella Research. J. Infect. Dis. 223, 187-188 (2021).

14. Tavares da Silva, L. B. et al. Molecular Verification of New World Mansonella perstans Parasitemias. Emerg. Infect. Dis. 23, 545-547 (2017).

15. Raccurt, C. P. Mansonella ozzardi and its vectors in the New World: an update with emphasis on the current situation in Haiti. J. Helminthol. 92, 655-661 (2018).

16. Calvopina, M., Chiluisa-Guacho, C., Toapanta, A., Fonseca, D. \& Villacres, I. High Prevalence of Mansonella ozzardi Infection in the Amazon Region, Ecuador. 25, (2019).

17. Kozek, W. J., Palma, G., Henao, A., García, H. \& Hoyos, M. Filariasis in Colombia: Prevalence and Distribution of Mansonella Ozzardi and Mansonella (=Dipetalonema) Perstans Infections in the Comisaría Del Guainía. Am. J. Trop. Med. Hyg. 32, 379-384 (1983).

18. Crainey, J. L. et al. Deep-sequencing reveals occult mansonellosis co-infections in residents from the Brazilian Amazon village of São Gabriel da Cachoeira. Clin. Infect. Dis. Off. Publ. Infect. Dis. Soc. Am. (2020) doi:10.1093/cid/ciaa082.

19. Langworthy, N. G. et al. Macrofilaricidal activity of tetracycline against the filarial nematode Onchocerca ochengi: elimination of Wolbachia precedes worm death and suggests a dependent relationship. Proc. R. Soc. Lond. B Biol. Sci. 267, 1063-1069 (2000).

20. Bazzocchi, C. et al. Combined ivermectin and doxycycline treatment has microfilaricidal and adulticidal activity against Dirofilaria immitis in experimentally infected dogs. Int. J. Parasitol. 38, $1401-1410$ (2008). 
21. Taylor, M. J. et al. Preclinical development of an oral anti-Wolbachia macrolide drug for the treatment of lymphatic filariasis and onchocerciasis. Sci. Transl. Med. 11, (2019).

22. Clare, R. H. et al. Industrial scale high-throughput screening delivers multiple fast acting macrofilaricides. Nat. Commun. 10, 11 (2019).

23. Hübner, M. P. et al. In vivo kinetics of Wolbachia depletion by ABBV-4083 in L. sigmodontis adult worms and microfilariae. PLoS Negl. Trop. Dis. 13, e0007636 (2019).

24. Hong, W. D. et al. AWZ1066S, a highly specific anti-Wolbachia drug candidate for a short-course treatment of filariasis. Proc. Natl. Acad. Sci. 116, 1414-1419 (2019).

25. Keiser, P. B. et al. Molecular identification of Wolbachia from the filarial nematode Mansonella perstans. Mol. Biochem. Parasitol. 160, 123-128 (2008).

26. Coulibaly, Y. I. et al. A Randomized Trial of Doxycycline for Mansonella perstans Infection. N. Engl. J. Med. 361, 1448-1458 (2009).

27. Debrah, L. B. et al. The Efficacy of Doxycycline Treatment on Mansonella perstans Infection: An Open-Label, Randomized Trial in Ghana. Am. J. Trop. Med. Hyg. 101, 84-92 (2019).

28. Casiraghi, M., Favia, G., Cancrini, G., Bartoloni, A. \& Bandi, C. Molecular identification of Wolbachia from the filarial nematode Mansonella ozzardi. Parasitol. Res. 87, 417-420 (2001).

29. Nikoh, N. et al. Evolutionary origin of insect-Wolbachia nutritional mutualism. Proc. Natl. Acad. Sci. 111, 10257-10262 (2014).

30. Hosokawa, T., Koga, R., Kikuchi, Y., Meng, X.-Y. \& Fukatsu, T. Wolbachia as a bacteriocyte-associated nutritional mutualist. Proc. Natl. Acad. Sci. 107, 769-774 (2010).

31. Grobusch, M. P., Kombila, M., Autenrieth, I., Mehlhorn, H. \& Kremsner, P. G. No evidence of Wolbachia endosymbiosis with Loa loa and Mansonella perstans. Parasitol. Res. 90, 405-408 (2003).

32. Gehringer, C. et al. Molecular Evidence of Wolbachia Endosymbiosis in Mansonella perstans in Gabon, Central Africa. J. Infect. Dis. 210, 1633-1638 (2014). 
33. Sandri, T. L. et al. Molecular Epidemiology of Mansonella Species in Gabon. J. Infect. Dis. 223, 287296 (2021).

34. Basano, S. de A. et al. Phase III Clinical Trial to Evaluate Ivermectin in the Reduction of Mansonella ozzardi infection in the Brazilian Amazon. Am. J. Trop. Med. Hyg. 98, 786-790 (2018).

35. Lima, N. F., Gonçalves-Lopes, R. M., Kruize, Y. C. M., Yazdanbakhsh, M. \& Ferreira, M. U. CD39 and immune regulation in a chronic helminth infection: The puzzling case of Mansonella ozzardi. PLoS Negl. Trop. Dis. 12, e0006327 (2018).

36. Ritter, M. et al. Mansonella perstans microfilaremic individuals are characterized by enhanced type 2 helper $\mathrm{T}$ and regulatory $\mathrm{T}$ and $\mathrm{B}$ cell subsets and dampened systemic innate and adaptive immune responses. PLoS Negl. Trop. Dis. 12, e0006184 (2018).

37. Poole, C. B. et al. In Silico Identification of Novel Biomarkers and Development of New Rapid Diagnostic Tests for the Filarial Parasites Mansonella perstans and Mansonella ozzardi. Sci. Rep. 9, 10275 (2019).

38. Nurk, S., Meleshko, D., Korobeynikov, A. \& Pevzner, P. A. metaSPAdes: a new versatile metagenomic assembler. Genome Res. 27, 824-834 (2017).

39. Langmead, B. \& Salzberg, S. L. Fast gapped-read alignment with Bowtie 2. Nat. Methods 9, 357-359 (2012).

40. Laetsch, D. R. \& Blaxter, M. L. BlobTools: Interrogation of genome assemblies. F1000Research 6, 1287 (2017).

41. Tatusova, T. et al. NCBI prokaryotic genome annotation pipeline. Nucleic Acids Res. 44, 6614-6624 (2016).

42. Li, H. Minimap2: pairwise alignment for nucleotide sequences. Bioinformatics 34, 3094-3100 (2018).

43. Marçais, G. et al. MUMmer4: A fast and versatile genome alignment system. PLOS Comput. Biol. 14, e1005944 (2018). 
44. Krzywinski, M. et al. Circos: An information aesthetic for comparative genomics. Genome Res. 19, 1639-1645 (2009).

45. Gu, Z., Gu, L., Eils, R., Schlesner, M. \& Brors, B. circlize Implements and enhances circular visualization in R. Bioinforma. Oxf. Engl. 30, 2811-2812 (2014).

46. Simão, F. A., Waterhouse, R. M., loannidis, P., Kriventseva, E. V. \& Zdobnov, E. M. BUSCO: assessing genome assembly and annotation completeness with single-copy orthologs. Bioinformatics 31, 3210-3212 (2015).

47. Yoon, S.-H., Ha, S., Lim, J., Kwon, S. \& Chun, J. A large-scale evaluation of algorithms to calculate average nucleotide identity. Antonie Van Leeuwenhoek 110, 1281-1286 (2017).

48. Meier-Kolthoff, J. P., Auch, A. F., Klenk, H.-P. \& Göker, M. Genome sequence-based species delimitation with confidence intervals and improved distance functions. BMC Bioinformatics 14, 60 (2013).

49. Emms, D. M. \& Kelly, S. OrthoFinder: phylogenetic orthology inference for comparative genomics. Genome Biol. 20, 238 (2019).

50. Lex, A., Gehlenborg, N., Strobelt, H., Vuillemot, R. \& Pfister, H. UpSet: Visualization of Intersecting Sets. IEEE Trans. Vis. Comput. Graph. 20, 1983-1992 (2014).

51. Conway, J. R., Lex, A., Gehlenborg, N. \& Hancock, J. UpSetR: an R package for the visualization of intersecting sets and their properties. Bioinformatics 33, 2938-2940 (2017).

52. Varani, A. M., Siguier, P., Gourbeyre, E., Charneau, V. \& Chandler, M. ISsaga is an ensemble of webbased methods for high throughput identification and semi-automatic annotation of insertion sequences in prokaryotic genomes. Genome Biol. 12, R30 (2011).

53. Arndt, D. et al. PHASTER: a better, faster version of the PHAST phage search tool. Nucleic Acids Res. 44, W16-W21 (2016). 
54. Akhter, S., Aziz, R. K. \& Edwards, R. A. PhiSpy: a novel algorithm for finding prophages in bacterial genomes that combines similarity- and composition-based strategies. Nucleic Acids Res. 40, e126e126 (2012)

55. Huerta-Cepas, J. et al. Fast Genome-Wide Functional Annotation through Orthology Assignment by eggNOG-Mapper. Mol. Biol. Evol. 34, 2115-2122 (2017).

56. Kanehisa, M., Furumichi, M., Tanabe, M., Sato, Y. \& Morishima, K. KEGG: new perspectives on genomes, pathways, diseases and drugs. Nucleic Acids Res. 45, D353-D361 (2017).

57. Sinha, A., Li, Z., Sun, L. \& Carlow, C. K. S. Complete Genome Sequence of the Wolbachia wAlbB Endosymbiont of Aedes albopictus. Genome Biol. Evol. 11, 706-720 (2019).

58. Lefoulon, E. et al. Large Enriched Fragment Targeted Sequencing (LEFT-SEQ) Applied to Capture of Wolbachia Genomes. Sci. Rep. 9, 1-10 (2019).

59. Basyoni, M. M. A. \& Rizk, E. M. A. Nematodes ultrastructure: complex systems and processes. J. Parasit. Dis. 40, 1130-1140 (2016).

60. Raverdy, S., Foster, J. M., Roopenian, E. \& Carlow, C. K. S. The Wolbachia endosymbiont of Brugia malayi has an active pyruvate phosphate dikinase. Mol. Biochem. Parasitol. 160, 163-166 (2008).

61. Andersson, S. G. E. \& Kurland, C. G. Reductive evolution of resident genomes. Trends Microbiol. 6, 263-268 (1998).

62. Foster, J. et al. The Wolbachia Genome of Brugia malayi: Endosymbiont Evolution within a Human Pathogenic Nematode. PLOS Biol. 3, e121 (2005).

63. Cotton, J. A. et al. The genome of Onchocerca volvulus, agent of river blindness. Nat. Microbiol. 2, 16216 (2016).

64. Kumar, S. \& Blaxter, M. L. Simultaneous genome sequencing of symbionts and their hosts. Symbiosis 55, 119-126 (2011). 
774 65. Kumar, S., Jones, M., Koutsovoulos, G., Clarke, M. \& Blaxter, M. Blobology: exploring raw genome

775 data for contaminants, symbionts, and parasites using taxon-annotated GC-coverage plots.

776 Bioinforma. Comput. Biol. 4, 237 (2013).

777 66. McGarry, H. F., Egerton, G. L. \& Taylor, M. J. Population dynamics of Wolbachia bacterial

778 endosymbionts in Brugia malayi. Mol. Biochem. Parasitol. 135, 57-67 (2004).

779 67. Mourembou, G. et al. Mansonella, including a Potential New Species, as Common Parasites in

780 Children in Gabon. PLoS Negl. Trop. Dis. 9, e0004155 (2015).

781 68. Cerveau, N., Leclercq, S., Leroy, E., Bouchon, D. \& Cordaux, R. Short- and Long-term Evolutionary

782 Dynamics of Bacterial Insertion Sequences: Insights from Wolbachia Endosymbionts. Genome Biol.

$783 \quad$ Evol. 3, 1175-1186 (2011).

784

785

786 
Figure $1:$ Jupiter plot showing high synteny between the de novo assembled $w$ Moz and wMpe genomes

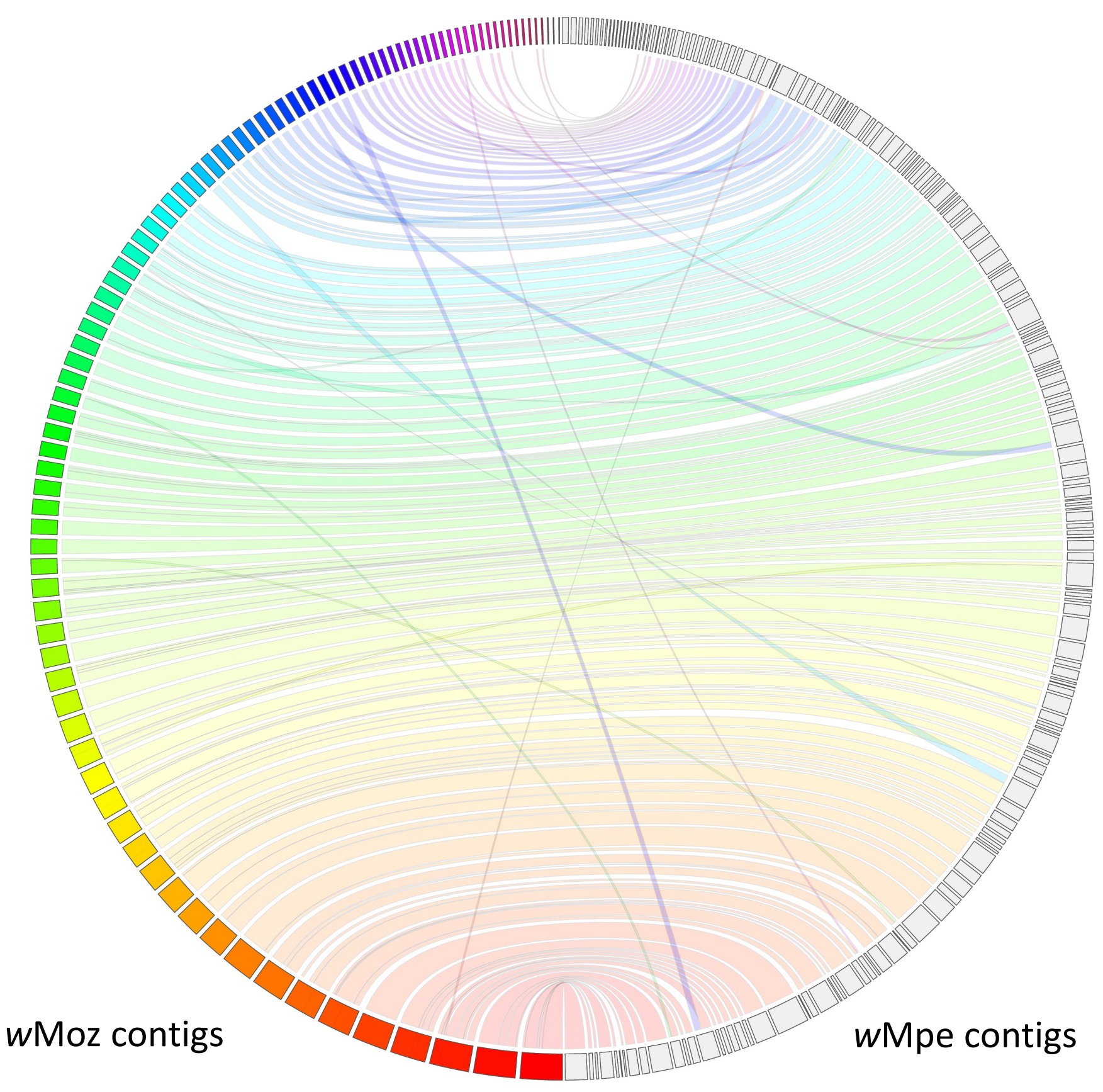


Figure 2 : Jupiter plots showing synteny between (A) wMoz and (B) wMpe with the wCle genome

(A)
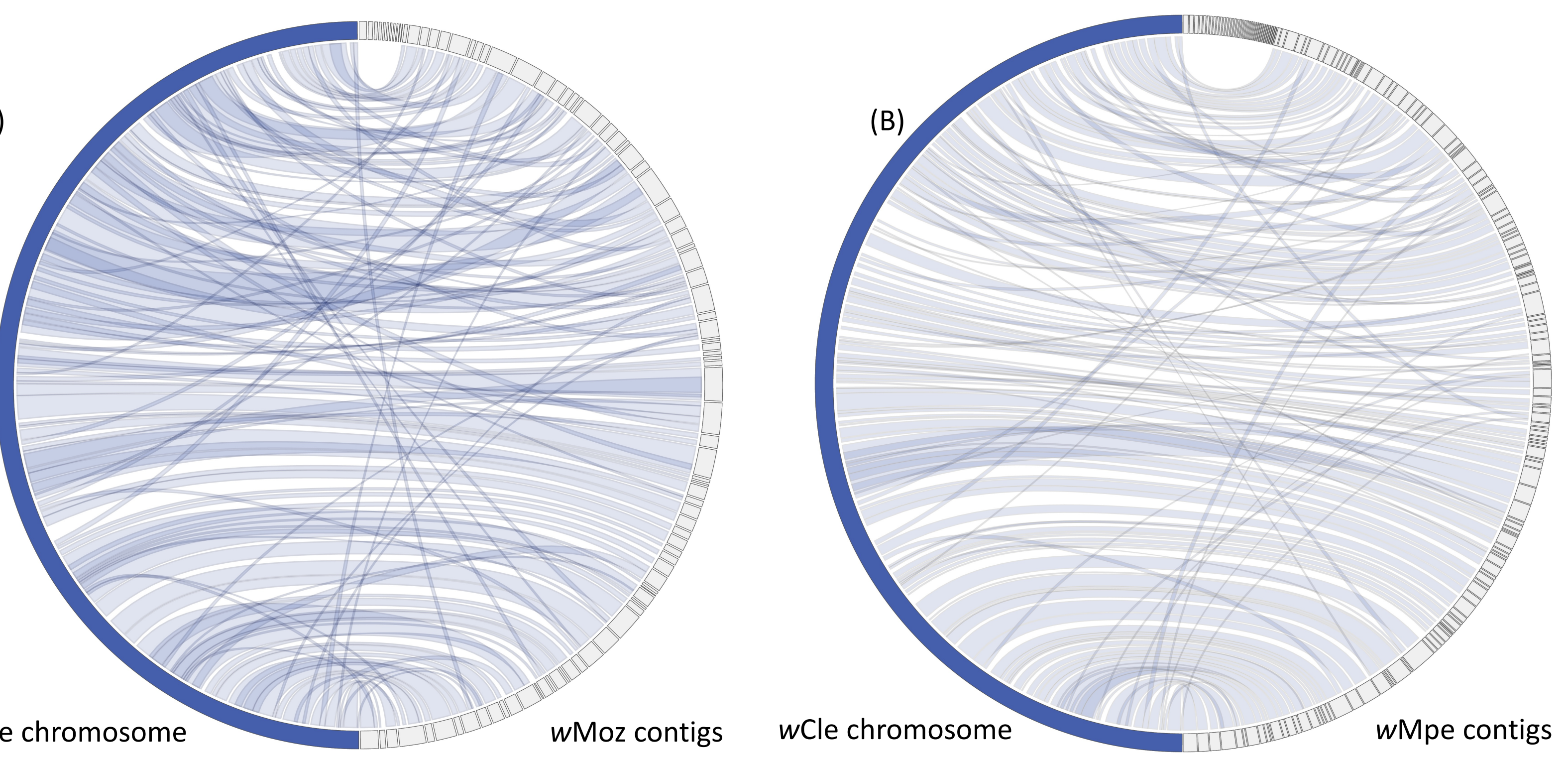


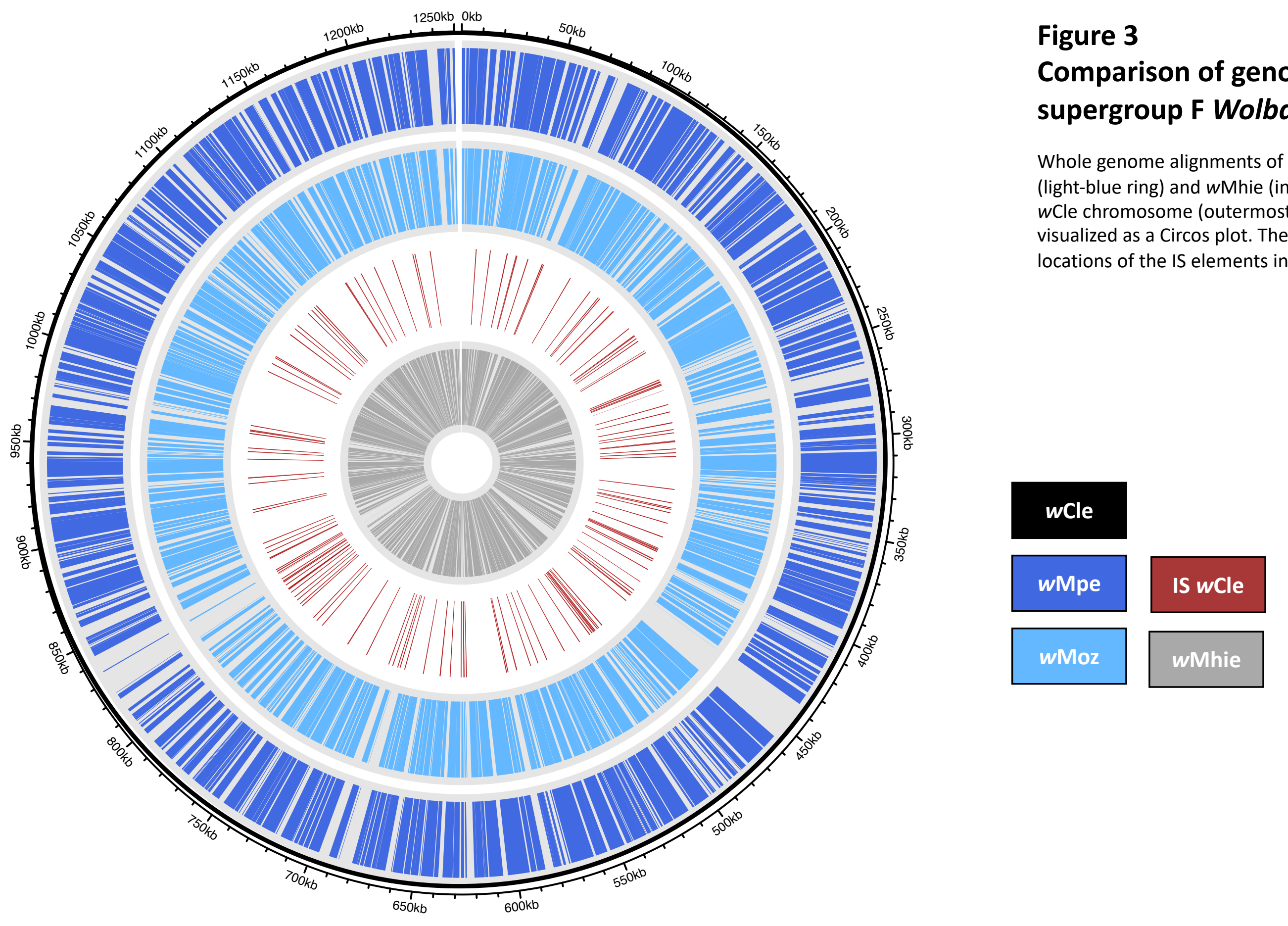


Figure 4: Global comparisons between multiple Wolbachia genomes using genome-wide Average Nucleotide Identity (gANI) scores, and digital DNA:DNA Hvbridization (dDDH) scores
(A) $\frac{0}{\frac{0}{3}}$
L $\quad$ WPpe ${ }_{100} \sum_{\xi}^{N}$

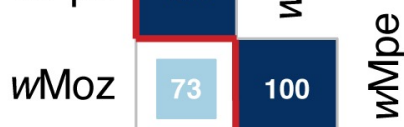
\begin{tabular}{l|l|l|l|l|l} 
WMpe & 73 & 97 & 100 & $\frac{\mathscr{\varrho}}{\varrho}$ \\
\hline
\end{tabular}
wCle

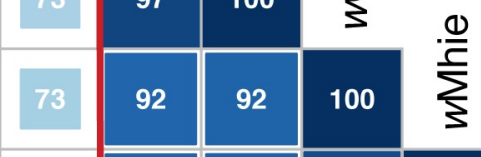
\begin{tabular}{l|l|l|l|l|l|l} 
WMhie & 73 & 92 & 92 & 95 & 100 & $\stackrel{0}{9}$ \\
\hline
\end{tabular}
wLsig
\begin{tabular}{|l|l|l|l|l|l|}
\hline 74 & 83 & 83 & 84 & 83 & 100 \\
引
\end{tabular}

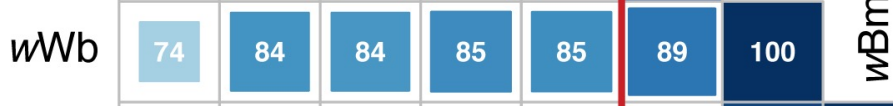

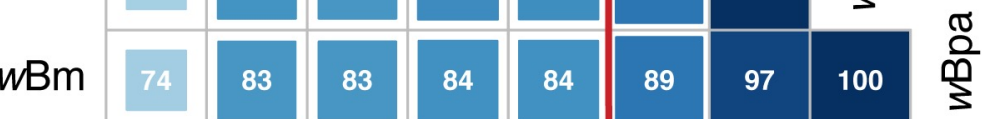
\begin{tabular}{l|l|l|l|l|l|l|l|l|l|l|}
\hline WBpa & 74 & 83 & 83 & 84 & 84 & 89 & 97 & 99 & 100 & ङ \\
\hline
\end{tabular}
\begin{tabular}{l|l|l|l|l|l|l|l|l|l|l|l|} 
WCtub & 73 & 80 & 80 & 81 & 80 & 80 & 80 & 80 & 80 & 100 & $\varrho$ \\
\hline
\end{tabular}

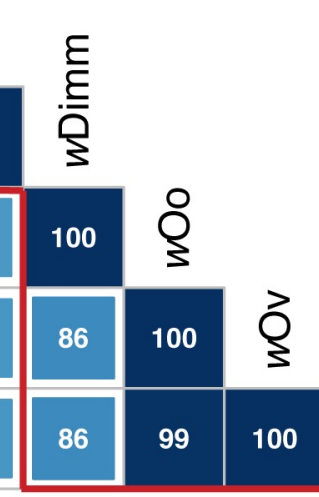
wov

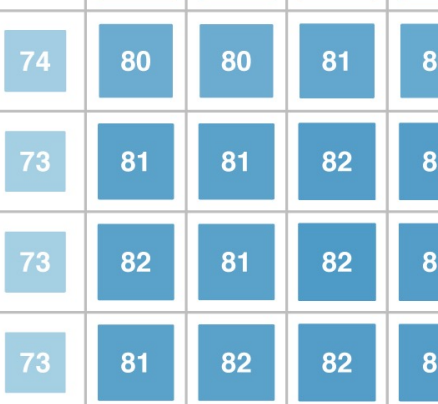
wDimm
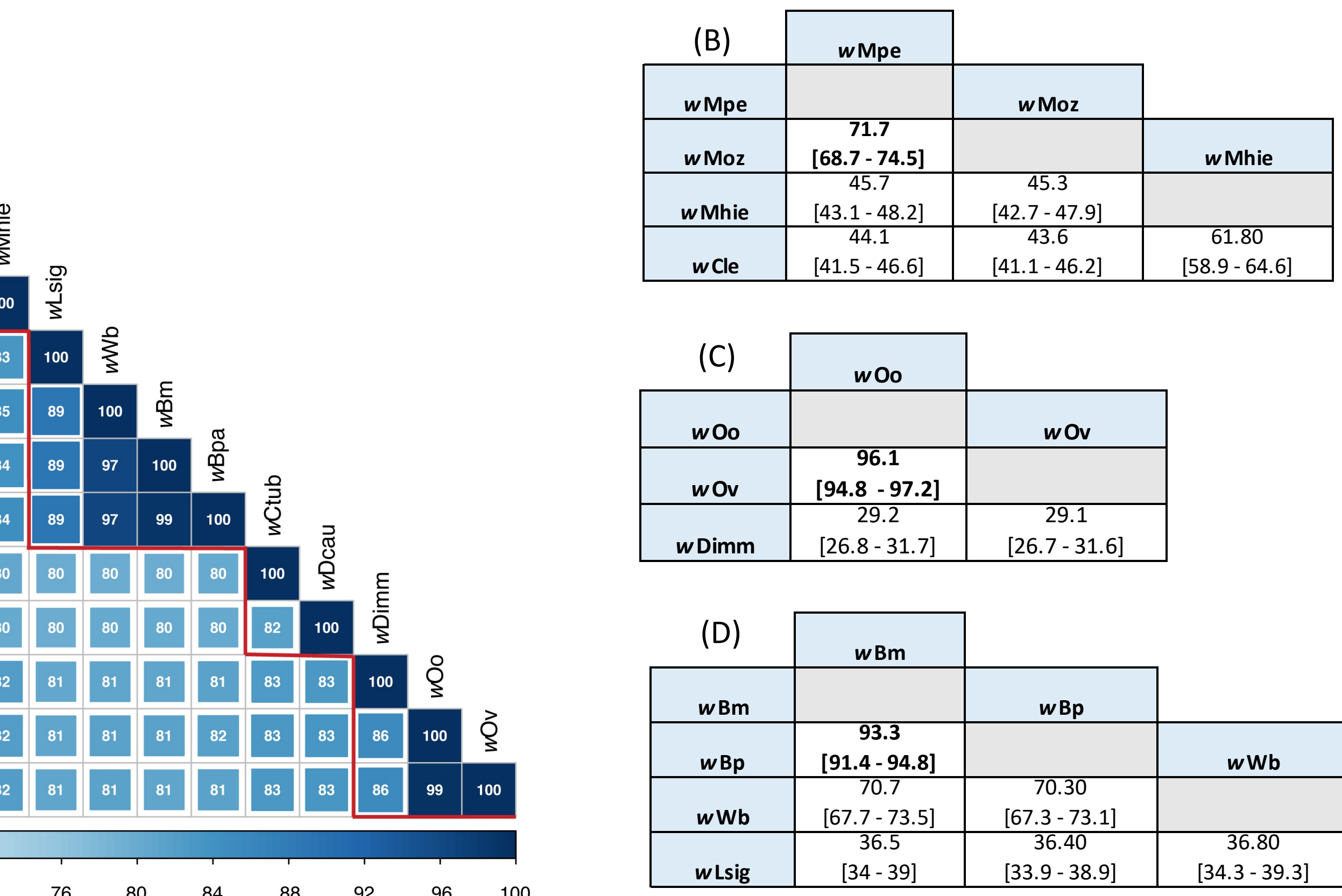
$64 \quad 68$
$72 \quad 76$
80
84 
Figure 5 :Orthogroups conserved across wMpe, wMoz compared to 18 other Wolbachia genomes
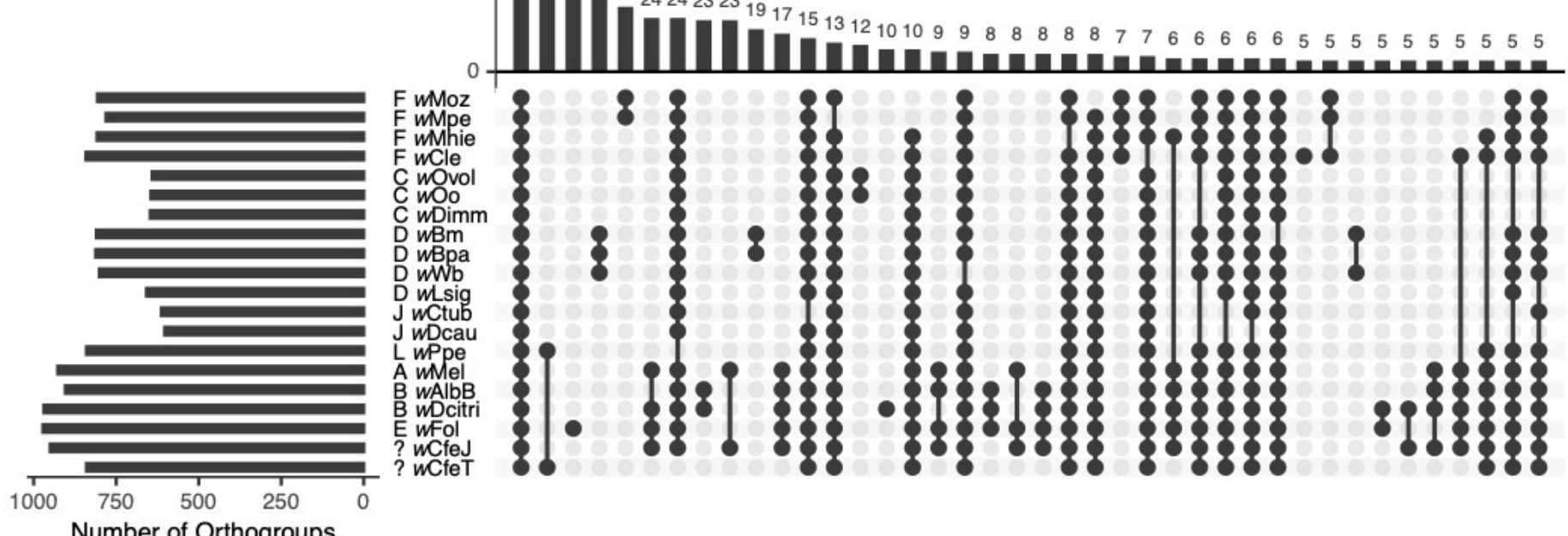
Figure 6. Venn diagram comparing pseudogenes in supergroup F Wolbachia

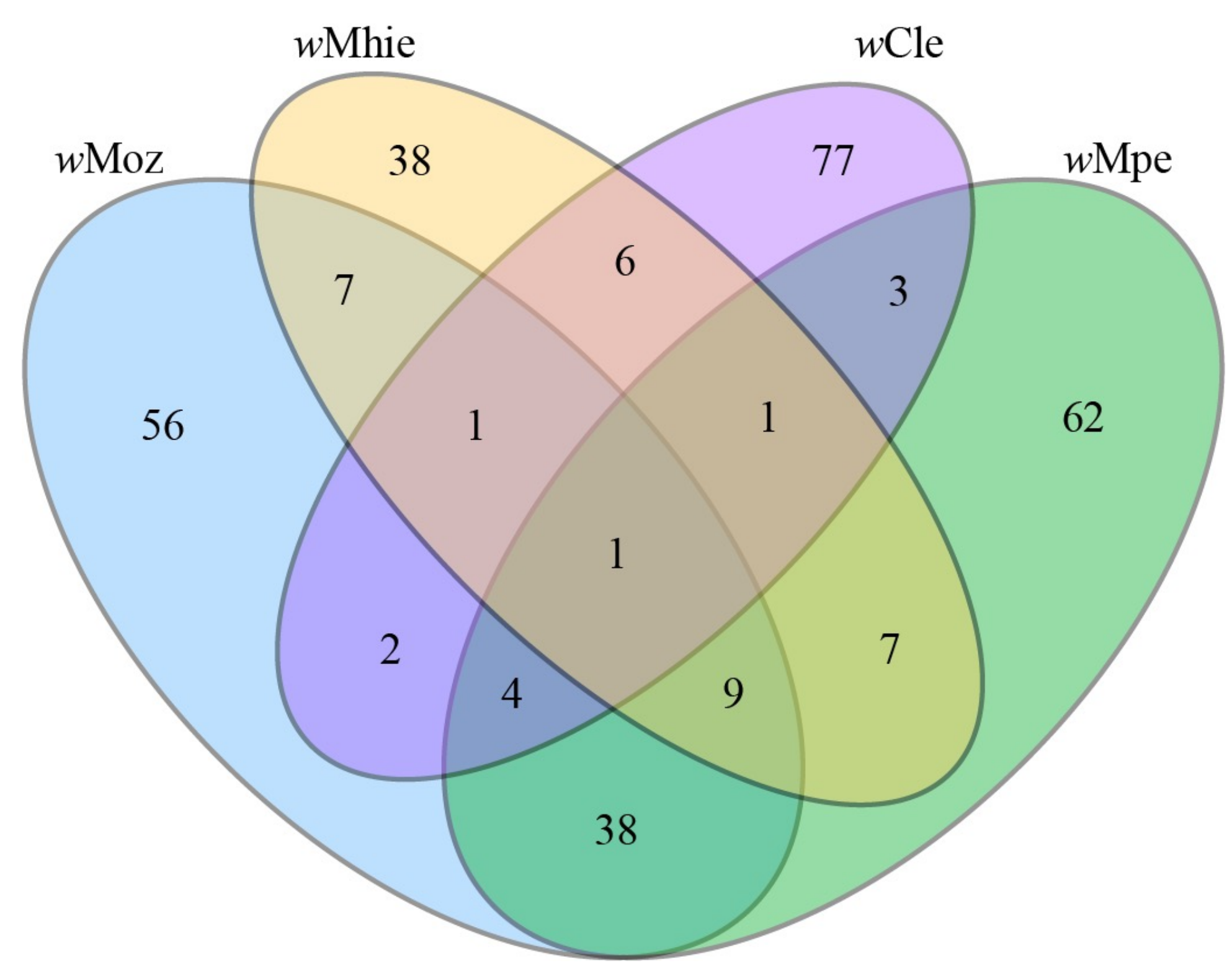




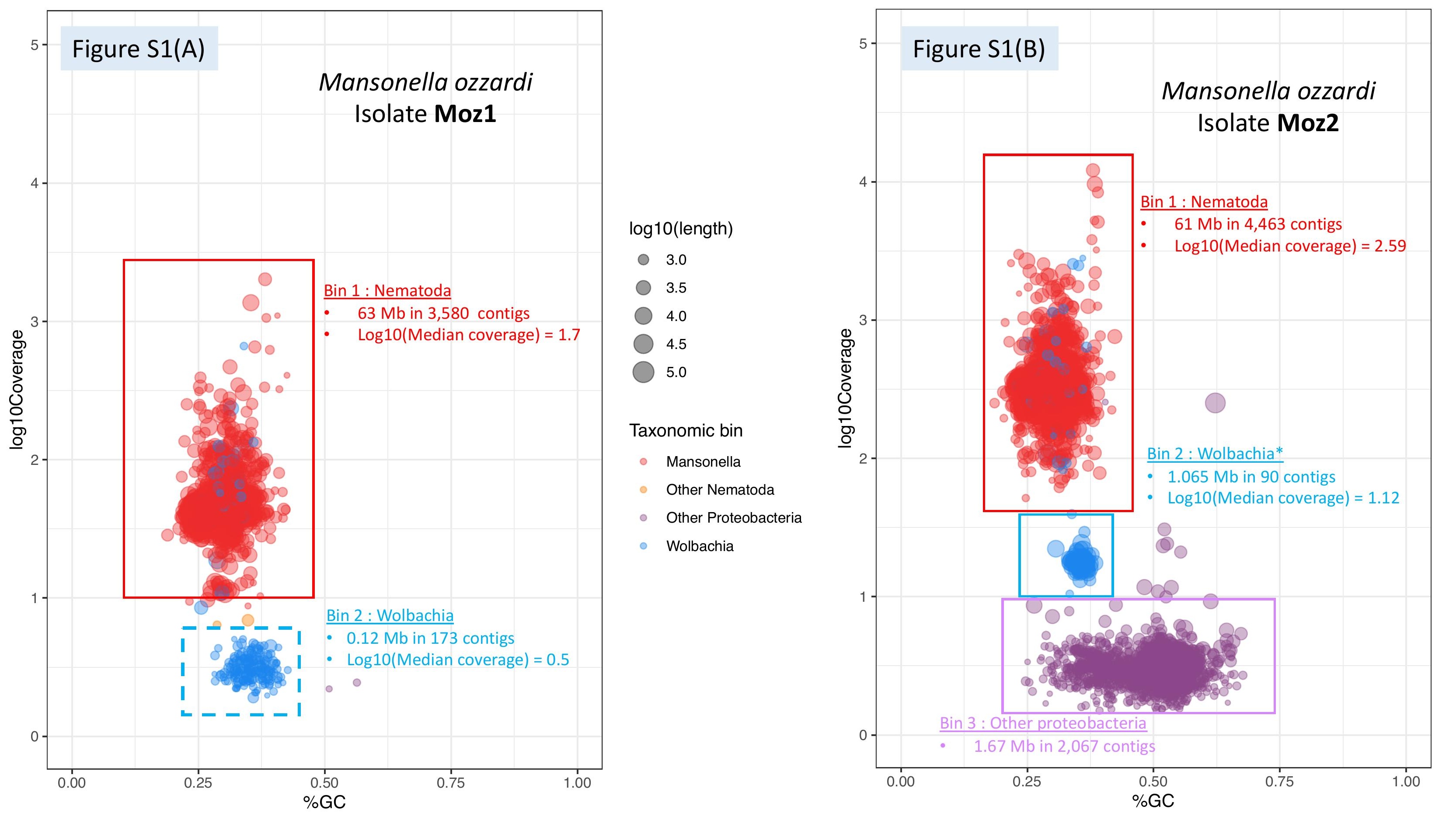


